\title{
Estudio de los Valores de Hemograma en Niños con Caries Temprana de la Infancia Severa
}

\author{
Study of Hemogram Values in Children with Severe Early Childhood Caries
}

Eugenia Henríquez- D’Aquino'; Sonia Echeverría López';

Silvia Espinosa Cereceda² \& Catalina Quintana Carvajal ${ }^{3}$

\begin{abstract}
HENRÍQUEZ- D'AQUINO, E.; ECHEVERRÍA, L. S.; ESPINOSA, C. S. \& QUINTANA, C. C. Estudio de los valores de hemograma en niños con caries temprana de la infancia severa. Int. J. Odontotomat., 13(4):452-457, 2019.

RESUMEN: La caries temprana de la infancia severa (CTI-S) es una enfermedad crónica que afecta a niños menores de 6 años, produce dolor, infección y destrucción de los tejidos dentales. El dolor que experimentan los niños con CTI-S puede llevar a hábitos alimenticios alterados que pueden causar deficiencias nutricionales. El objetivo fue evaluar los valores de hemograma en niños con CTI-S y compararlos con los valores normales de referencia para la edad. Se realizó un estudio retrospectivo observacional. Se analizaron las fichas y hemogramas de 47 niños con CTI-S, clasificados como ASA 1, atendidos bajo anestesia general en el Hospital de la Fuerza Aérea de Chile. Se tomaron en cuenta los valores del hemograma en relación a: Hematocrito, Hemoglobina y VCM. Se realizaron test descriptivos para las variables en estudio y se utilizó el testt para comparar los valores de hemograma con los valores normales de referencia. Se encontró una disminución de los valores de hematocrito en 4 pacientes (8,5 \%) y una disminución del valor de VCM en 17 pacientes (36,7 \%). Se encontraron diferencias significativas al comparar los promedios obtenidos en relación a hematocrito, VCM y hemoglobina en niños con CTI-S con el promedio de referencia $(p<0,001)$. De acuerdo con los resultados obtenidos en este estudio, podemos concluir que los niños con caries temprana de la infancia severa, tienen alteraciones en los valores promedio de hemograma en relación a hematocrito, hemoglobina y VCM.
\end{abstract}

PALABRAS CLAVE: caries temprana de la infancia severa, valores de hemograma, niños preescolares.

\section{INTRODUCCIÓN}

La Caries Temprana de la Infancia severa (CTIS) es una forma única de caries que se desarrolla en la dentición primaria inmediatamente después de erupcionados los primeros dientes en infantes y preescolares. (AAPD, 2016) Hay numerosos factores de riesgo asociados a esta enfermedad, biológicos, conductuales y sociales que contribuyen al proceso de caries (Mahesh et al., 2013). Es una enfermedad que progresa rápidamente y que se caracteriza por presentar dolor, infección y destrucción total de los dientes primarios en sus etapas más severas (Hallet \& O’Rourke, 2006). Sus consecuencias a largo plazo pueden incluir alteraciones en la calidad de vida, en el crecimiento y desarrollo y malnutrición (Clarke et al., 2006; Naidu et al., 2016).

Los niños con CTI-S tienen un alto riesgo de tener un menor desarrollo físico especialmente peso y talla, que puede ser causado por problemas para comer debido a dolor en sus dientes primarios (Thomas \& Primosch, 2002; Echeverría et al., 2009; Gaur \& Nayak, 2011). El dolor dentario continuo e insoportable, tiene serios efectos que pueden ser irreversibles en los niños pequeños (Echeverría et al., 2010; Shamsaddin et al., 2017). Los niños con CTI-S, la mayoría de las veces, tienen caries no tratadas que producen dificultad para masticar ciertos alimentos que son ricos en hierro y vitamina $\mathrm{C}$ como carnes rojas y frutas cítricas o tienen una dieta con altos contenidos de azúcares que puede afectar el consumo de otros nutrientes (Tang et al., 2013). Estos hábitos alimentarios alterados pueden causar deficiencias nutricionales (Clarke et al.; Shaoul et al., 2012; Schroth et al., 2013), niños con CTI-S han sido significativamente asociados con anemia por deficiencia de hierro (Organización Mundial de la Salud, 2011; Naidu

\footnotetext{
${ }^{1}$ Departamento del Niño y Ortopedia Dento-Maxilar, Facultad Odontología, Universidad de Chile, Santiago, Chile.

${ }^{2}$ Hospital fuerza Aérea de Chile. Facultad Odontología, Universidad de Chile, Santiago, Chile.

${ }^{3}$ Cirujano-Dentista. Ejercicio Privado, Santiago, Chile.
} 
et al.; Nur et al., 2016). La reducción de los niveles de hemoglobina es una ocurrencia común en muchas enfermedades crónicas (Shamsaddin et al.), la caries temprana de la infancia es una enfermedad crónica, que puede afectar severamente a los niños, los cuales sufren de inflamación debido a pulpitis y abscesos dentarios que afectan la eritropoyesis (Schroth et al.). Una de las consecuencias importantes inducidas por la CTI-S, es la malnutrición, siendo esta caries, un posible factor de riesgo para la anemia por déficit de hierro (Colak et al., 2013; Hashemi et al., 2018).

Una forma de diagnosticar estas deficiencias, es mediante un hemograma, según como se vean alterados los valores normales de cada uno de sus índices (Becker, 2001). Aunque, algunos estudios han mostrado que los niños con CTI-S pueden tener alteraciones en diferentes índices hematológicos (Shamsaddin et al; Hashemi et al.), se sabe poco acerca de la influencia de esta enfermedad en el hemograma, por lo tanto, el objetivo de este estudio, fue evaluar en el hemograma de niños con caries temprana de la infancia severa los valores de hematocrito, hemoglobina y VCM y compararlos con valores de referencia normales para la edad.

\section{MATERIAL Y MÉTODO}

Se realizó un estudio observacional, retrospectivo de corte transversal, aprobado para su realización por el Comité de Ética del Hospital de la Fuerza Aérea de Chile y el Comité de Ética de la Facultad de Odontología de la Universidad de Chile ( Proyecto PRI-ODO 1001). La muestra estuvo constituida por las fichas clínicas y sus respectivos hemogramas preoperatorios, de niños menores de 6 años con CTI$\mathrm{S}$ atendidos bajo anestesia general en el Hospital de la Fuerza Aérea de Chile entre los años 2007 y 2012. Se analizaron un total de 123 fichas clínicas de las cuales 47 cumplieron con los criterios de inclusión, los cuales fueron: niños menores de 6 años con CTI$\mathrm{S}$, clasificados como ASA I, fichas clínicas con su hemograma completo y consentimiento informado.

En base a los antecedentes consignados en la ficha odontológica de cada paciente, caries temprana de la infancia severa, en el presente estudio fue considerada de acuerdo a la definición aceptada por la Academia Americana de Pediatría Dental (American Academy of Pediatric Dentistry, 2016) que indica que en los niños menores de 3 años, cualquier signo de caries en superficies lisas es indicativo de CTI-S. Entre los 3 y 5 años, una o más superficies cavitadas, perdidas por caries u obturadas en los incisivos superiores; o un índice ceo-s $\geq 4$ (a los 3 años), $\geq 5$ (a los 4 años), $0 \geq 6$ (a los 5 años) constituye CTI-S.

Los valores de hemograma tomados en cuenta fueron: hematocrito $(\mathrm{Hct})$, hemoglobina $(\mathrm{Hgb})$ y volumen corpuscular medio (VCM), los cuales fueron comparados con los valores de referencia para niños entre 2 y 12 años de edad del Ministerio de Salud (Ministerio de Salud, 2018), considerándose un rango normal de hematocrito entre 35 y $45 \%$; hemoglobina entre 11 y $16 \mathrm{~g} / \mathrm{dL}$ y VCM entre 77 y $95 \mathrm{fL}$.

Para el análisis de los resultados, se realizó la estadística descriptiva de las variables medidas, en base al cálculo de frecuencias y medidas de resumen. Para determinar si existieron diferencias estadistícamente significativas entre los valores promedio de los hemogramas estudiados con respecto a los valores promedio de referencia, se utilizó el test-t.

\section{RESULTADOS}

Se analizaron 47 fichas clínicas y 47 hemogramas de niños con CTI-S. La muestra se caracterizó por estar constituida por un $59,6 \%$ de niños de género masculino y un $40,4 \%$ de género femenino, cuyo promedio de edad fue de 47,6 \pm 11 meses, con un índice ceod promedio de 9,6 $\pm 3,3$ dentro de los cuales un $68 \%$ de los niños presentaba compromiso pulpar en sus dientes primarios.

En la Tabla I, se muestran los promedios de los valores de hematocrito, hemoglobina y VCM observados en la muestra. La comparación de los valores de hematocrito con respecto a los valores de referencia, se representan en la Figura 1. La mayoría de los niños mostraron valores de hematocrito dentro de los valores normales, solo 4 casos $(8,5 \%)$ presentaron un valor más bajo de lo normal. En la Figura 2, se representa la comparación de los valores de hemograma en relación a hemoglobina. Los resultados obtenidos muestran que todos los niños estudiados tuvieron valores normales de hemoglobina. En relación a la comparación de los valores de VCM con los valores normales, se observa que 17 casos $(36,7$ $\%$ ) presentaron valores bajo la norma (Fig. 3). 
Tabla I. Distribución de los valores de hemograma en niños con caries temprana de la infancia severa.

\begin{tabular}{lcccc}
\hline Parámetro & N total & Valor mayor & Valor menor & Promedio (DS) \\
\hline Hematocrito (\%) & 47 & 42,1 & 32,3 & $37,4 \pm 2,3$ \\
Hemoglobina (gr/dL) & 47 & 15 & 11 & $12,9 \pm 0,8$ \\
VCM (fL) & 47 & 89,2 & 69 & $78,5 \pm 3,7$ \\
\hline
\end{tabular}
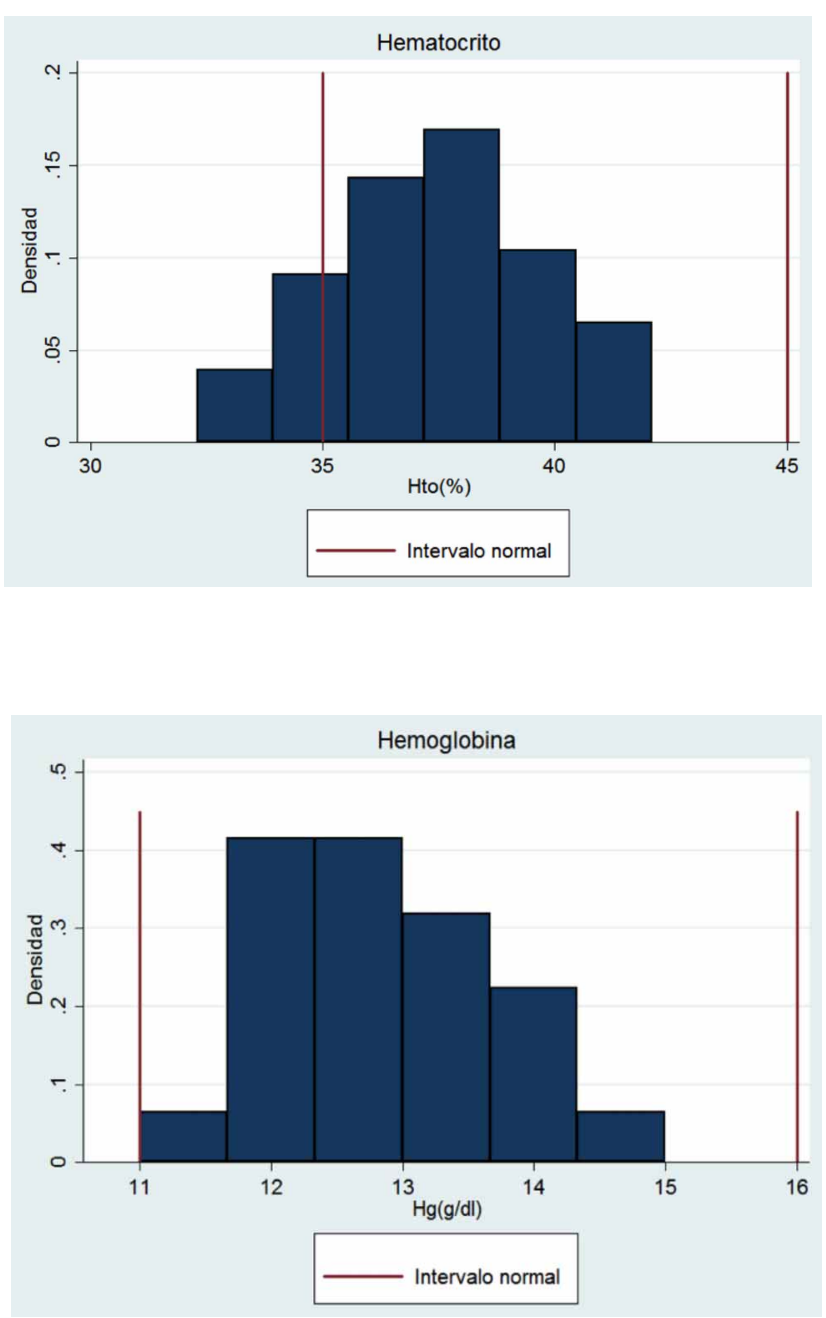

Para determinar si había diferencias significativas entre los valores de hemograma de los niños estudiados y los valores de referencia, se utilizó el test-t. Se compararon los promedios del grupo para cada parámetro sanguíneo analizado con los promedios

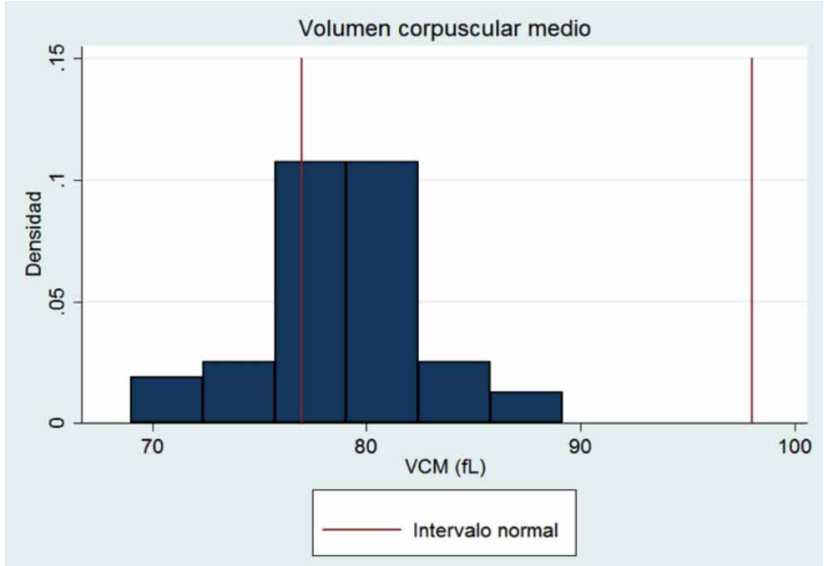

normales, los resultados obtenidos mostraron diferencias significativas al comparar la media obtenida con la media de referencia. En la Tabla II, se observa que los promedios de los parámetros evaluados fueron significativamente menores que los promedios de referencia $(p<0,001)$.

Con el propósito de determinar una posible relación de las variables género, ceod y compromiso pulpar con las alteraciones en los valores de hemograma obtenidos, se utilizó el Test exacto de Fisher, no encontrándose diferencias significativas en ninguno de los parámetros estudiados: género $(p=$ $0,121)$; ceod $(p=0,789)$ y compromiso pulpar $(p=0,817)$

\section{DISCUSIÓN}

Una consecuencia de la caries temprana de la infancia severa es la malnutrición que puede producir anemia. Este estudio constituye un aporte para deter-

Tabla II. Comparación entre valores promedio de hemograma en niños con CTI-S y valores promedio de referencia normal.

\begin{tabular}{lccc}
\hline Parámetro & Promedio & Promedio de referencia* $^{*}$ & ${\text { Valor } P^{* *}}^{*}$ \\
\hline Hematocrito $(\%)$ & 37,4 & 40 & $<0,001$ \\
Hemoglobina (gr/dL) & 12,9 & 13,5 & $<0,001$ \\
VCM (fL) & 78,5 & 86 & $<0,001$ \\
\hline
\end{tabular}


minar las alteraciones producidas en el hemograma por esta enfermedad y aunque el número de pacientes estudiados es bajo, representa la experiencia de un hospital público, donde se realiza atención de niños con CTI-S bajo anestesia general y a los cuales por protocolo se les solicita un hemograma preoperatorio. Esta investigación se enfocó en evaluar los valores de hemograma en relación a hematocrito, hemoglobina y VCM, que son reconocidos índices de anemia por déficit de hierro (Nur et al.) que es la causa más común de anemia en niños (Organización Mundial de la Salud). Los resultados obtenidos revelaron diferencias significativas entre los valores promedio de hemograma obtenidos y los valores promedio de referencia, los niños con caries temprana de la infancia severa tenían valores promedio más bajos de hematocrito, hemoglobina y VCM.

La investigación de parámetros sanguíneos y su relación con caries es escasa y a nivel nacional, no hay estudios publicados con los cuales se puedan contrastar los resultados obtenidos. En este estudio se encontró que un $8,5 \%$ de la muestra presentaba valores de hematocrito más bajos que los normales. Este resultado es parecido a los resultados reportados por el estudio de Tang et al., en niños con CTI-S en el cual un $14 \%$ de niños presentaba valores más bajos a lo normal de hematocrito (Tang et al.), pero difieren de los resultados de otro estudio en el cual los investigadores reportaron niveles normales de hematocrito en niños con CTI-S (Nur et al.). En relación a los niveles de hemoglobina, a diferencia de otros estudios que reportaron niveles significativamente bajos en los niños estudiados (Clarke et al.; Tang et al.; Schroth et al.; Bansal et al., 2016), los valores encontrados por nuestra investigación se encontraron dentro de los rangos normales. Estos resultados están en concordancia con los hallazgos encontrados por Nur et al, los cuales reportaron que los niños con CTI-S tenían valores normales de hemoglobina. Al contrario, al comparar el promedio de hemoglobina del grupo con la media de referencia, se encontró que los valores obtenidos en este estudio eran significativamente menores, esto se explicaría debido a la distribución de los valores en la muestra en la cual más de la mitad de estos valores (29/47) se agrupan entre 11 y $13(\mathrm{~g} / \mathrm{dL})$ y ningún valor se distribuyó entre 15 y $16(\mathrm{~g} / \mathrm{dL})$, esto sugiere que es la distribución de los valores obtenidos y no la presencia de valores bajo la norma, lo que hace que exista diferencias significativas entre el promedio obtenido y el promedio de referencia para los valores de hemoglobina. Cuando se analizó el valor del VCM, se encontró que este valor estaba alterado en un 36,7
$\%$ de los pacientes estudiados. Este resultado muestra una alteración mayor a la encontrada en trabajos anteriores, pero está en relación con otros estudios que encontraron al igual que esta investigación casi un $40 \%$ de los niños con CTI-S tenían valores de VCM más bajos (Bansal et al.; Nur et al.).

Un valor de VCM disminuido es sugerente de anemia microcitica, la cual es una característica distintiva observada en la anemia por déficit de hierro (Shaoul et al.). Con los hemogramas analizados, no se puede determinar la causa exacta de la microcitosis encontrada en los niños estudiados. Una hipótesis podría ser que esta disminución en el tamaño de los eritrocitos este dada por una ingesta inadecuada de hierro, causa más común de anemia en niños, y se podría explicar por la presencia de dolor y dificultades para masticar alimentos fibrosos como carnes y verduras crudas que se observa en los niños cuando existe dolor asociado a la CTI-S (Echeverría et al., 2010). Otra explicación puede estar dada por el hecho de que la absorción de hierro es influenciada por la dieta, los niños con CTI-S a menudo tienen un excesivo consumo de leche y el exceso de calcio disminuye la absorción de hierro (Shaoul et al.; Tang et al.).

Se ha demostrado una significante relación entre CTI-S y bajos niveles de hierro, pero el mecanismo por el cual se produce, es desconocido, una hipótesis sugerida por Gaur \& Nayak indica que una disminución de los niveles de hemoglobina puede ser asociado a una respuesta inflamatoria del cuerpo que podría resultar en la producción de citoquinas que inhiben la síntesis de eritrocitos disminuyendo la producción de hemoglobina en la sangre y por lo tanto el nivel de hierro (Gaur \& Nayak). En este estudio un $68 \%$ de los niños tuvieron compromiso pulpar, lo cual indica la presencia de inflamación en sus dientes primarios.

Según la Organización Mundial de la Salud, se debe considerar anemia por déficit de hierro, cuando al menos 2 de 3 parámetros (Ferritina sérica, Hemoglobina y VCM) se encuentran más bajo que lo normal. De acuerdo a los resultados obtenidos en este estudio no se puede afirmar que los niños estudiados presenten anemia ya que solo el VCM presentó valores disminuidos. De acuerdo al Ministerio de Salud (Hertrampf et al., 2009), la prevalencia de anemia en Chile es muy baja (0-3\%) gracias a los programas nutricionales llevados a cabo por esta entidad, esto podría explicar la diferencia existente con los valores obtenidos con los estudios anteriormente mencionados que mostraron bajos niveles de hemoglobina. 
Las limitaciones de este estudio recaen en el tamaño muestral que es muy pequeño, basado en una muestra por conveniencia de los pacientes con caries extremadamente severas, atendidos bajo anestesia general, que no permitió contar con un grupo control de niños sin caries, y además por las características del diseño de la investigación, el cual no permite determinar causalidad, por lo que no podemos precisar con exactitud si la CTI-S es responsable de las alteraciones en el hemograma encontradas en estos pacientes.

De acuerdo a los resultados obtenidos y a las limitaciones, se puede concluir que los niños con caries temprana de la infancia severa estudiados presentan alteraciones en sus hemogramas en relación a hematocrito, hemoglobina y VCM. Las implicancias clínicas de estos hallazgos son importantes, tanto para los odontopediatras como para los odontólogos que atienden niños, y este estudio puede ser tomado como un piloto o un estudio preliminar, que nos alerta que aunque la anemia no se ha establecido, estos niños pueden estar en riesgo de padecerla y por lo tanto sería necesario derivar a estos niños al pediatra para realizar estudios más profundos al respecto que incluyan el estudio de los niveles de hierro en estos pacientes.

\section{AGRADECIMIENTOS}

Los autores expresan su agradecimiento al Hospital de la Fuerza Aérea de Chile por su apoyo a la gestión del estudio.

HENRÍQUEZ- D'AQUINO, E.; ECHEVERRÍA, L. S.; ESPINOSA, C. S. \& QUINTANA, C. C. Study of hemogram values in children with severe early childhood caries. Int. J. Odontostomat., 13(4): 452-457, 2019.

ABSTRACT: Severe early childhood caries (S-ECC) is a multifactorial chronic disease that affects children under 6 years of age, produces pain, infection and destruction of the dental tissues. The pain experienced by children with SECC may lead to altered eating habits that may cause nutritional deficiencies. The aim of this study was to evaluate the hemogram values in children with severe early childhood caries, and compare them with age population reference values. An observational retrospective study was carried out. We analyzed the medical records and their respective hemograms of 47 children with S-ECC, classified as ASA1, attended at the Chilean Air Force Hospital under general anesthesia. The hemogram values were taken into account in relation to: hematocrit, hemoglobin and mean corpuscular volume (MCV). Descriptive tests were carried out for the variables under study and the t-test was used to compare the hemogram values with the normal reference values. A decrease in hematocrit values was found in 4 patients $(8.5$ $\%)$ and a decrease in the value of MCV in 17 patients (36.7 $\%)$. Significant differences were found when comparing the averages obtained in relation to hematocrit, hemoglobin and MCV in children with S-ECC with the reference average ( $p$ $<0.001$ ). According to the results obtained, in this study, we can conclude that children with severe early childhood caries, have alterations in the average of hemogram values in relation to hematocrit, hemoglobin and MCV.

KEY WORDS: severe early childhood caries, hemogram values, preschool.

\section{REFERENCIAS BIBLIOGRÁFICAS}

American Academy of Pediatric Dentistry. Policy on Early Childhood Caries (ECC): Classifications, Consequences, and Preventive Strategies. Pediatr. Dent., 38(6):52-4, 2016.

Bansal, K.; Goyal, M. \& Dhingra, R. Association of severe early childhood caries with iron deficiency anemia. J. Indian Soc. Pedod. Prev. Dent., 34(1):36-42, 2016.

Becker, K. A. Interpretación del hemograma. Rev. Chil. Pediatr., 72(5):460-5, 2001.

Clarke, M.; Locker, D.; Berall, G.; Pencharz, P.; Kenny, D. J. \& Judd, $P$. Malnourishment in a population of young children with severe early childhood caries. Pediatr. Dent., 28(3):254-9, 2006.

Colak, H.; Dülgergil, C. T.; Dalli, M. \& Hamidi, M. M. Early childhood caries update: A review of causes, diagnoses, and treatments. J. Nat. Sci. Biol. Med., 4(1):29-38, 2013.

Echeverría, L. S.; Henríquez D’Aquino, E. \& Linacre, S. D. Peso y talla en niños con caries temprana de la infancia. Estudio comparativo. Rev. Dent. Chile, 100(1):25-30, 2009.

Echeverría, L. S.; Henríquez D`Aquino, E.; Sepúlveda, R. \& Barra, P. M. Caries temprana de la infancia severa: Impacto en la calidad de vida relacionada a la salud oral de niños preescolares. Rev. Dent. Chile, 101(2):15-21, 2010.

Gaur, S. \& Nayak, R. Underweight in low socioeconomic status preschool children with severe early childhood caries. J. Indian Soc. Pedod. Prev. Dent., 29(4):305-9, 2011.

Hallet, K. B. \& O'Rourke, P. K. Pattern and severity of early childhood caries. Community Dent. Oral Epidemiol., 34(1):25-35, 2006.

Hashemi, A.; Bahrololoomi, Z. \& Salarian, S. Relationship Between Early Childhood Caries and Anemia: A Systematic Review. Iran J. Ped. Hematol. Oncol., 8(2):126-38, 2018.

Hertrampf, E.; Olivares, M.; Brito, A. \& Castillo-Carniglia, A. Evaluación de la Prevalencia de Anemia Ferropriva en una Muestra Representativa de la Región Metropolitana y Quinta Región de los Beneficiarios del Programa Nacional de Alimentación Complementaria (PNAC). Santiago de Chile, Ministerio de Salud, 2009. Disponible en: https://www.minsal.cl/portal/url/item/ 94a33f151 efca574e04001011f0131dd.pdf

Mahesh, R.; Muthu, M. \& Rodrigues, S. J. Risk factors for early childhood caries: a case-control study. Eur. Arch. Paediatr. Dent., 14(5):331-7, 2013.

Ministerio de Salud (MINSAL). Guía de Interpretación del Hemograma. Santiago de Chile, Hospital de Niños Roberto del Río, Servicio de Salud Metropolitano Norte, Ministerio de Salud, Gobierno de Chile, 2018. Disponible en: http://www.ssmn.cl/des- 
HENRÍQUEZ- D'AQUINO, E.; ECHEVERRÍA, L. S.; ESPINOSA, C. S. \& QUINTANA, C. C. Estudio de los valores de hemograma en niños con caries temprana de la infancia severa. Int. J. Odontotomat., 13(4):452-457, 2019

cargas/protocolos_referencia_contrareferencial hospital_roberto_del_rio/hematooncologia/hemogramas.pdf

Naidu, R.; Nunn, J. \& Donnelly-Swift, E. Oral health-related quality of life and early childhood caries among preschool children in Trinidad. BMC Oral Health, 16(1):128, 2016

Nur, B. G.; Tanrıver, M.; Altunsoy, M.; Atabay, T. \& Intepe, N. The prevalence of iron deficiency anemia in children with severe early childhood caries undergoing dental surgery under general anesthesia. Pediatr. Dent. J., 26(2):83-7, 2016.

Organización Mundial de la Salud (OMS). Concentraciones de Hemoglobina para Diagnosticar la Anemia y Evaluar su Gravedad. Ginebra, Organización Mundial de la Salud, 2011. Disponible en: http://www.who.int/vmnis/indicators/haemoglobin_es.pdf

Schroth, R. J.; Levi, J.; Kliewer, E.; Friel, J. \& Moffat, M. E. K. Association between iron status, iron deficiency anaemia, and severe early childhood caries: a case-control study. BMC Pediatr., 13:22, 2013.

Shamsaddin, H.; Jahanimoghadam, F.; Poureslami, H. \& Haghdoost, A. A. The association between growth factors and blood factors with early childhood caries. J. Oral Health Oral Epidemiol., 6(4):196-202, 2017.

Shaoul, R.; Gaitini, L.; Kharouba, J.; Darawshi, G.; Maor, I. \& Somri, $M$. The association of childhood iron deficiency anaemia with severe dental caries. Acta Paediatr., 101(2):e76-9, 2012.

Tang, R. S.; Huang, M. C. \& Huang, S. T. Relationship between dental caries status and anemia in children with severe early childhood caries. Kaohsiung J. Med. Sci., 29(6):330-6, 2013.

Thomas, C. W. \& Primosch, R. E. Changes in incremental weight and well-being of children with rampant caries following complete dental rehabilitation. Pediatr. Dent., 24(2):109-13, 2002.
Dirección para correspondencia:

Eugenia Henríquez D’Aquino

Departamento del Niño y Ortopedia Dento-Maxilar

Facultad Odontología

Universidad de Chile

Santiago

CHILE

Email: eugeniahd@gmail.com

Recibido : 28-04-2019

Aceptado: 02-07-2019 